\title{
Impacto de la pandemia por COVID-19 en la salud mental
}

\author{
The impact of the COVID-19 \\ pandemic on mental health
}

\author{
Impacto da pandemia da \\ COVID-19 na Saúde Mental
}

El riesgo de contagio por el virus del SARS-CoV-2, causante de la COVID-19, inició en el mundo a finales del 2019 en Hubei, China. Para el momento de esta edición existían más de 176,480,226 personas diagnosticadas con la enfermedad y 3,825,240 fallecidas en el mundo. En México, se reporta una Tasa de Fatalidad por Caso del 9.4\% (CFR por sus siglas en inglés)'.

Referente a la COVID-19, revisiones sistemáticas en la literatura internacional y meta análisis han señalado la prevalencia de otra pandemia asociada a la COVID-19, se trata de los riesgos a la salud mental por el uso de sustancias psicoactivas y las diversas condiciones neurológicas actuales. De acuerdo con Lancet ${ }^{2}$, 17\% de 116 países habían referido la necesidad de atender tales condiciones de riesgo relacionadas con la aparición de la COVID-19, desde mediados del 2020.

Se tenía registro de antecedentes en el mundo respecto a la elevada asociación entre síndromes respiratorios graves durante las emergencias sanitarias por el SARS, el Ébola y las condiciones de riesgo a la salud mental. Entre $33 \%$ y $42 \%$ de las personas admitidas en los hospitales por SARS o Síndrome Respiratorio de Oriente Medio (MERS) padecieron depresión, ansiedad, daños de memoria e insomnio, entre otros riesgos a la salud mental, al parecer directamente derivados de la propia infección por tales virus ${ }^{3}$.

Sin embargo, independientemente de las secuelas neurológicas derivabas de la infección, los determinantes sociales juegan un papel relevante en la aparición subsecuente de síntomas y riesgos a la salud mental. No menos importante es el uso de sustancias psicoactivas de forma exponencial y explosiva, así como las condiciones de violencia física y emocional, que parecen también incrementar durante las emergencias sanitarias como medios de afrontamiento en las personas.

Considérese también que en los países de nivel socioeconómico medio bajo es donde se muestran las prevalencias más altas de trastornos mentales por uso de sustancias y condiciones neurológicas graves, tanto previamente, como durante y después de las emergencias sanitarias 3 . En dichos estados 
mentales se presentan proporciones altas de personas con depresión, insomnio, ansiedad, irritabilidad, daño en la memoria, fatiga, memoria traumática, desórdenes del sueño, estrés postraumático, así como riesgo de autolesión y suicidio4.

Dado el contexto de amenazas de muerte, pérdidas de los seres queridos y cercanos, las dificultades económicas como académicas derivadas de la pandemia y el requerido distanciamiento social; ha resultado necesario establecer estrategias de prevención, atención y seguimiento de tales condiciones de riesgo a la salud mental, por el consumo de sustancias psicoactivas y condiciones neurológicas en México, como en el mundo.

Ante la pandemia por COVID-19, el conjunto de condiciones de riesgo a la salud mental a corto plazo incluye situaciones de estrés agudo emocional característicos de una respuesta adaptativa y de preparación natural de las personas ante los riesgos de perder la vida. Asimismo, incluye las condiciones traumáticas asociadas a la emergencia sanitaria debido a los fallecimientos y la imposibilidad de establecer ritos funerarios, los cuales cumplen un papel fundamental en la aceptación y transición de las pérdidas.

Sin embargo, dichos entornos de estrés natural y adaptativo pueden llegar a derivar en condiciones psicológicas de corte conductual, emocional y cognitivo que rebasan las capacidades de las personas para enfrentar las emergencias; ocasionando pérdida de apetito, fatiga, insomnio, irritabilidad, problemas de atención, así como miedo. Tales condiciones combinadas con el uso de sustancias psicoactivas o estar inmerso en un contexto de violencia pueden derivar en problemas graves de salud mental, a corto, mediano y largo plazo5.

Las condiciones de riesgo a la salud mental no solamente derivan del propio padecimiento de la enfermedad por COVID-193, sino también de los determinantes sociales que deben abordarse por los equipos multidisciplinarios para la salud integral. La atención por los efectos del distanciamiento5,

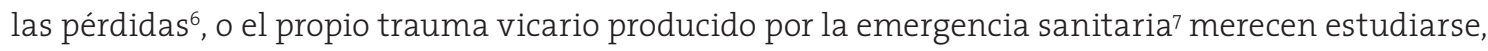
tanto en la fase de alerta, como en las de resistencia y agotamiento sugeridas por Selye ${ }^{8}$ ante los eventos traumáticos como los que representa la pandemia por COVID-19.

A pesar de contar con solo 10 profesionales de la salud por cada 100 mil habitantes en el mundo9 y 3 por cada 100 mil habitantes en México $^{10}$, resulta fundamental mantener acciones concretas para enfrentar eventos como los ya descritos, entre los que destacan: la ansiedad, la depresión y el enojo $0^{11,12}$.

En México, Morales et al., ${ }^{6}$ describieron síntomas importantes de atender, asociados con el diagnóstico por COVID-19, las pérdidas de seres queridos por esta enfermedad o bien por el diagnóstico de depresión previo a contraer COVID-19. Tales autores encontraron una alta asociación con estrés agudo, ansiedad generalizada, tristeza y enojo en dichas condiciones. Recientemente, Morales et al. ${ }^{7}$ reportaron índices de evitación, tristeza, distanciamiento, enojo y ansiedad como efecto del estrés agudo. También reportaron altos índices de ansiedad generalizada como efecto del miedo a perder la salud. De forma relevante se reportó un incremento del uso explosivo del alcohol durante la cuarentena, así como episodios de abuso físico y emocional, todo ello consecuentemente ligado a las condiciones de riesgo a la salud mental mencionadas.

En México como en el mundo, la ansiedad por el estado de salud se ha asociado fuertemente con índices de estrés agudo, evitación, distanciamiento, enojo y tristeza durante la pandemia. Parece ser que el distanciamiento reportado por las personas durante el confinamiento se ha asociado con niveles altos de síntomas de salud mental comparado con aquellas que reportan estar en confinamiento parcial o no estar en dichas condiciones. El consumo de alcohol, la violencia física y emocional también es mayor en estas condiciones aislamiento. 
En México, los síntomas de trastornos mentales también son más frecuentes cuando se reporta consumo y violencia física o emocional7. Los hallazgos recientes parecen indicar que, a partir del mes de confinamiento, se presentaron síntomas de la fase de resistencia descrita por Selye ${ }^{8}$, caracterizados por estrés agudo que pueden llegar a alcanzar condiciones de estrés postraumático, propios de la fase de agotamiento, los cuales deben prevenirse y atenderse en las etapas tempranas del padecimiento ${ }^{13}$.

En concreto, las condiciones de riesgo a la salud mental asociadas con el miedo a enfermarse o haber padecido COVID-19, sufrir pérdidas de seres cercanos y queridos, sufrir previamente de enfermedades emocionales, el distanciamiento social, así como las condiciones económicas constituyen amenazas y eventos traumáticos que han perdurado por más de 18 meses y que representan una necesidad inminente de atención por parte de los profesionales de la salud.

Tales profesionales se encuentran enfrentando una pandemia paralela, ya existente en el mundo, pero que ahora avanza a pasos agigantados hacia condiciones más inexorables como la alta incidencia de estrés postraumático, depresión severa, trastornos por uso de sustancias, autolesión y suicidio. Considérese también los trastornos neurológicos graves que merecen atención en todos los niveles: comunitario, de primer nivel, de segundo orden y hasta el servicio especializado en el tercer nivel de atención.

Los riesgos a la salud mental por consumo de sustancias y enfermedades neurológicas establecen necesidades de atención para reducir la morbilidad asociada a sus condiciones emocionales, cognitivas y conductuales. Por consiguiente, resulta fundamental desarrollar estrategias basadas en la evidencia para atender dichos riesgos ${ }^{14}$ e intervenir de forma temprana y efectiva ${ }^{15}$. Es importante generar e instrumentar líneas de investigación que permitan identificar las condiciones eficientes de prevención, de atención de los determinantes sociales de mayor relevancia, así como de los efectos directos de la infección por COVID-19 sobre las condiciones neurológicas que explican la presencia de condiciones de salud mental como la depresión o la ansiedad ${ }^{3}$. Asimismo, es necesario evaluar el efecto de la implementación de las intervenciones psicosociales remotas, para todos los niveles y ordenes de riesgo que se han venido implementando en México como en el mundo?.

De esta manera, las políticas públicas locales, estatales y nacionales deberán derivarse de los hallazgos y de la evidencia científica e incrementar las probabilidades exitosas de escalamiento y mantenimiento de tales acciones efectivas5. La política pública efectiva permitirá reducir significativamente los riesgos de perder la salud mental en las poblaciones de bajos recursos y niveles socioeconómicos, donde ocurren con mayor prevalencia.

\author{
S. Morales-Chainé \\ ORCID: 0000-0001-9269-7877 \\ Universidad Nacional Autónoma de México, \\ Facultad de Psicología, Ciudad de México, México \\ smchaine@gmail.com
}

\title{
REFERENCIAS
}

1. Pan American Health Organization/World Health Organization. Region of the Americas Update. Geneva: PAHO/WHO; 2021. https://bit.ly/3BJtk56

2. The Lancet Infectious Diseases. The intersection of COVID-19 and mental health. Lancet Infect Dis. 2020; 2O(11): 1217. https://doi.org/10.1016/S1473-3099(20)30797-0 
3. Rogers JP, Chesney E, Oliver D, Pollak TA, McGuire P, Fusar-Poli P, et al. Psychiatric and neuropsychiatric presentations associated with severe coronavirus infections: A systematic review and meta-analysis with comparison to the COVID-19 pandemic. Lancet Psychiatry. 2020; 7(7): 611-27.

https://doi.org/10.1016/S2215-0366(20)30203-0

4. Salari N, Hosseinian-Far A, Jalali R, Vaisi-Raygani A, Rasoulpoor S, Mohammadi M, et al. Prevalence of stress, anxiety, depression among the general population during the COVI-19 pandemic: A systematic review and meta-analysis. Global Health. 2020; 16(57): 1-11.

https://doi.org/10.1186/s12992-020-00589-w

5. Li Z, Ge J, Yang M, Feng J, Qiao M, Jiang R, et al. Vicarious traumatization in the general public, members, and non-members of medical teams aiding in COVID-19 control. Brain Behav Immun. 2020; 88: 916-9. https://doi.org/10.1016/j.bbi.2020.03.007

6. Morales-Chainé S, López-Montoya A, Bosch-Maldonado A, Beristain-Aguirre A, Robles-García R, López-Rosales F, et al. Condiciones de salud mental durante la pandemia por COVID-19. Rev. int. investig. Adicciones. 2020; 6(2): 11-24. https://doi.org/10.28931/riiad.2020.2.03

7. Morales-Chainé S, López-Montoya A, Bosch-Maldonado A, Beristain-Aguirre A, Robles-García R, Garibay-Rubio CR, et al. Mental health symptoms, binge drinking, and the experience of abuse during the COVID-19 lockdown in Mexico. Front. Public Health. 2021; 9(656036): 1-12. https://doi.org/10.3389/fpubh.2021.656036

8. Selye H. The Stress of Life. New York: McGraw-Hill; 1956.

9. World Health Organization. Mental health atlas. Geneva: WHO; 2014. https://bit.ly/zeYpues

10. Heinz G, Chapa GC, Carmona-Huerta J. Speciality in psychiatry: Mexico 2106. Salud ment. 2016; 39(2); 69-76. https://doi.org/10.17711/SM.0185-3325.2016.003

11. Li S, Wang Y, Xue J, Zhao N, Zhu T. The impact of COVID-19 epidemic declaration on psychological consequences: A study on active Weibo users. Int J Environ Res Public Health. 2020; 17(6): 1-9. https://doi.org/10.339o/ijerph17062032

12. Ho CS, Chee CY, Ho RC. Mental health strategies to combat the psychological impact of coronavirus disease 2019 (COVID-19) beyond paranoia and panic. Ann Acad Med Singap. 2020; 49(3): 155-60. https://bit.ly/zizlRor

13. Blevins CA, Weathers FW, Davis MT, Witte TK, Domino JL. The posttraumatic stress disorder checklist for DSM-5 (PCL-5): Development and initial psychometric evaluation. J Trauma Stress. 2015; 28(6): 489-98. https://doi.org/10.1002/jts.22059

14. Wang C, Pan R, Wan X, Tan Y, Xu L, Ho CS, et al. Immediate psychological responses and associated factors during the initial stage of the 2019 coronavirus disease (COVID-19) epidemic among the general population in China. Int J Environ Res Public Health. 2020; 17(5): 1-25. https://doi.org/10.339o/ijerph17051729

15. Zhue Y, Chen L, Ji H, Xi M, Fang Y, Li Y. The risk and prevention of novel coronavirus pneumonia infections among inpatients in psychiatric hospitals. Neurosci Bull. 2020; 36(3): 299-302.

https://doi.org/10.1007/s12264-020-00476-9 